\title{
A Parametric Study of Mechanical Cross-Coupling in Parallel-Kinematics Piezo-Flexural Nano-Positioning Systems
}

\author{
Abbas Shafiee ${ }^{*}{ }^{(1)}$, Amirhossein Ahmadian"\# ${ }^{(0)}$, Amin Akbari1\# \\ ${ }^{1}$ School of Mechanical Engineering, Purdue University, West Lafayette, USA \\ ${ }^{2}$ Department of Mechanical and Aerospace Engineering, University of California, Los Angeles, USA \\ Email: ^shafiee@purdue.edu, aakbari@purdue.edu, aahmadian@ucla.edu
}

How to cite this paper: Shafiee, A., Ahmadian, A. and Akbari, A. (2021) A Parametric Study of Mechanical Cross-Coupling in Parallel-Kinematics Piezo-Flexural NanoPositioning Systems. Open Journal of Applied Sciences, 11, 596-613.

https://doi.org/10.4236/ojapps.2021.115043

Received: April 28, 2021

Accepted: May 25, 2021

Published: May 28, 2021

Copyright $\odot 2021$ by author(s) and Scientific Research Publishing Inc. This work is licensed under the Creative Commons Attribution International License (CC BY 4.0).

http://creativecommons.org/licenses/by/4.0/

(c) (i) Open Access

\begin{abstract}
Piezo-electric nano-positioning stages are being widely used in applications in which precision and accuracy in the order of nano, and high scanning speeds are paramount. This paper presents a Finite Element Analysis (FEA) of the parallel piezo-flexural nano-positioning (PPNP) stages to investigate motion interference between their different axes. Cross-coupling is one of the significant contributors to undesirable runouts in the precision positioning of PPNP actuators. Using ABAQUS/CAE 2018 software, a 3D model of a PPNP stage was developed. The model consists of a central elastic body connected to a fixed frame through four flexural hinges. A cylindrical stack of multiple piezoelectric disks is placed between the moving central body and the fixed frame. Extensive simulations were carried out for three different friction coefficients in the piezoelectric disks' contact surfaces, different frame materials, and different geometrical configurations of the stage and the hinges. As a result, it was observed that the primary root cause of the mechanical crosscoupling effect could be realized in the combination of the slip and rotation of the piezoelectric disks due to their frictional behavior with the stage moving in the tangential direction, concurrent with changes in the geometry of the stage.
\end{abstract}

\section{Keywords}

Nano-Positioning Stages, Piezoelectric Actuators, Mechanical

Cross-Coupling, Finite Element Analysis, Friction

\section{Introduction}

Parallel piezo-flexural nano-positioning (PPNP) stages are used in a variety of ${ }^{\star}$ Corresponding Author.

\#The second and third authors have the same contribution. 
advanced technologies such as precision metrology, scanning probe microscopy [1]-[7], energy harvesting [8] [9] [10] [11], medical robotics [12] [13], biomechanics research apparatus [14]-[20], orthodontics [21] [22] [23], material [24] [25] [26], structural engineering [27] [28], microfluidic devices [29] [30] [31] [32] [33] and other instruments which need precise actuation. Several different factors limit the precision positioning of PPNP stages, including mechanical vibrations, external disturbances, hysteresis and creep effects, temperature variation, and the cross-coupling of the different axes. Understanding the underlying mechanism of cross-coupling in the PPNP stages is the main focus of this effort. In this work, a finite element model for a double-axis PPNP stage is developed to investigate this problem and propose potential solutions for its mitigation.

The nano-positioning systems' literature embodies a number of finite element analysis (FEA) efforts to understand the mechanics of piezo-flexural actuators. Elmustafa and Lagally [34] conducted an FEA to examine the flexural hinge behavior with controlled motion in a nano-positioning stage used for precision machining. They changed the stage's dimensions and realized that the natural frequency, displacement, stress, and the applied force to the hinges could be controlled and optimized for more convenient and accurate positioning of the stage. Sun et al. [35] designed a 2 DOF nano-positioning system and analyzed it through FEA. Their results show that the excitation force, stiffness, and the natural frequency of the system would increase by decreasing the length and increasing the hinges' width. $\mathrm{Li}$ and $\mathrm{Xu}$ [36] presented the process of designing and manufacturing a nano-positioning platform with compound parallelogram flexures and bridge-type displacement amplifiers. They derived analytical models for the mechanical performance evaluation of the stage in terms of stiffness, load capacity, kinematics, and dynamics and verified their results through FEA. Shi et al. [37] designed a novel parallel kinematic XY positioner with the large stroke, decoupled motions, compact size, and large out-of-plane stiffness. The three parts of the positioner are the thermal actuator, displacement amplifier, and a guidance mechanism. They showed that an asymmetrical parallel guide mechanism could be implemented to reduce the cross error in the $\mathrm{x}$ and $\mathrm{y}$ directions while increasing the out-of-plane stiffness. Nagel et al. [38] predicted the dual-stage, three-axis hybrid parallel-serial-kinematic nano-positioner's parasitic motion by introducing a new parallel-kinematic mechanism that can eliminate the effects of planar coupling. Their results also indicated the potential of the non-orthogonal mechanical amplifier design in minimizing the widespread cross-coupling nonlinearity of the parallel-kinematic designs. Zhang et al. [39] suggested a distributed multi-channel Hammerstein model, composed of a cascaded connection of a static nonlinearity and dynamic linearity to approximate the nonlinear spatial/temporal cross-coupling hysteresis. To reduce the crosscoupling hysteresis nonlinearities' adverse effect, Kang et al. [40] proposed a new modeling and control method for a six-axis parallel piezo-flexural micropositioning stage for bio micromanipulation application. A novel frictional- 
order normalized Bouc-Wen (FONBW) model was utilized to characterize the nonlinear hysteresis of the stage in conjugation with the rigid-flexible coupling dynamic model. They also developed an inverse FONBW-based hysteresis compensator with a decentralized control model to enhance the motion tracking performance by making the multi-input multi-output (MIMO) system decoupled.

The literature lacks investigating the effects of Friction between the piezoelectric disks and the stacks and the frame in piezo-flexural systems. One of this study's main motivations is to investigate the effects of frictional behavior on the mechanical cross-coupling effect in PPNP stages. A comprehensive model of a PPNP stage was simulated in ABAQUS finite element software, containing the model's central elastic body, which is movable in the center, connected to a fixed frame through four flexural hinges. In this model, seven separate cylindrical piezoelectric disks, one upon the other, are placed between the moving central body and the fixed frame. Simulations for various friction coefficients in the contact surfaces of the piezoelectric disks, different frame materials, and different perturbations in the stage's geometry and the hinges were carried out.

The remainder of the paper is organized as follows: In Section 2, the experimental observation of the cross-coupling effect in the PPNP stage from Bashash et al. [41] is reviewed. Section 3 presents model development and finite element analysis of the stage. Section 4 presents the FEA results and discussions. Finally, Section 5 summarizes the paper's concluding remarks.

\section{Materials and Methods}

\subsection{Cross-Coupling Effect in Piezo-Flexural Stages}

According to Bashash et al., the coupling of perpendicular axes in the PPNP stages imposes a significant limitation on their positioning accuracy [42]. Figure 1 shows a double-axis Physic Instrument (PI) piezo-flexural stage with two piezoelectric stack actuators and two capacitive position sensors [42]. By applying a voltage to one of the piezoelectric stacks, the stage moves along the stack's expansion/contraction direction. However, due to flexural coupling between the axes, a small portion of the motion is transferred to the perpendicular direction, inducing an undesirable motion in the other axis.

An earlier study has shown by moving the stage in one direction; the corresponding position sensor picks a slight displacement in the other direction [42]. In Figure 2, the PI stage's cross-coupling effect for two low and high-frequency sinusoidal inputs with the magnitude of $80 \mathrm{~V}$ applied to axis $\mathrm{x}$ of the PI stage, with no voltage applied to axis $y$, can be seen. The resultant motions are about $75 \mu \mathrm{m}$ for axis $\mathrm{x}$ and about $0.22 \mu \mathrm{m}$ for axis $\mathrm{y}$. This coupling includes a hysteresis effect and excites the system's natural frequency if the excitation frequency is high, e.g., $50 \mathrm{~Hz}$. Although the cross-coupling magnitude is shown as small as about $0.3 \%$, its impact on nanoscale positioning can be detrimental [42]. Hence, it is essential to take a step beyond the traditional control-oriented analysis of the cross-coupling effect and understand its underlying mechanics to minimize 
its undesirable impacts potentially. In the remainder of this effort, it focuses on the finite element analysis of the PI stage's cross-coupling effect, where the finite element model is corroborating with Bashash's work [42].

(a)

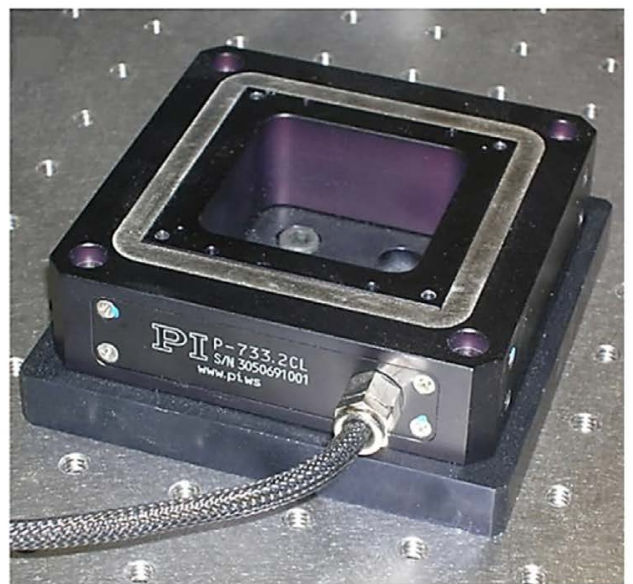

(b)

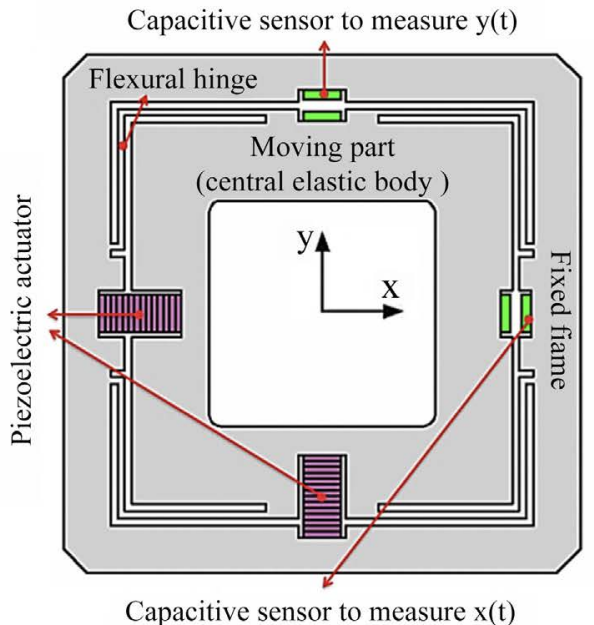

Figure 1. Experimental platform: (a) Physic Instrument P-733.2CL double-axis PPNP stage, and (b) its internal configuration and kinematics [42].

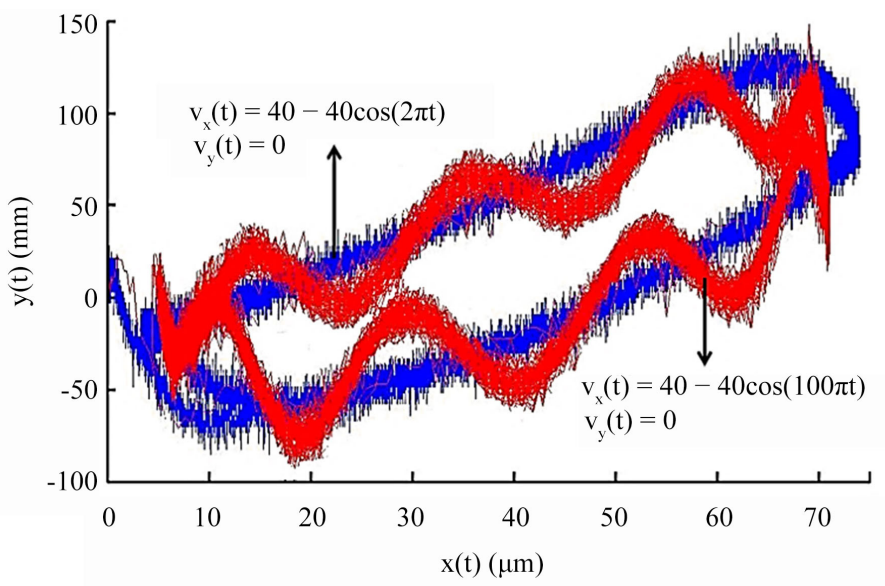

Figure 2. Motion interferences from axis $\mathrm{x}$ to axis $\mathrm{y}$ at $1 \mathrm{~Hz}$ and $50 \mathrm{~Hz}$ [42]. 


\subsection{Finite Element Analysis (FEA)}

In this paper, ABAQUS FEA software is used to carry out the process of mechanical cross-coupling simulation. In the simulation, the loading steps are modeled based on the closed-curve of the experimental data. This curve is mainly due to hysteresis and is modeled virtually in this study.

Due to the piezoelectric actuators' asymmetrical arrangements, the simulation is applied in a 3D geometry instead of the 2D geometry. Figure 3 and Figure 4 show the dimensions of the frame and the cylindrical piezoelectric disks, respectively.

Table 1 shows the mechanical properties of the frame and the piezoelectric disk. In all simulations, seven cylindrical piezoelectric elements have been used, as shown and numbered in Figure 5.

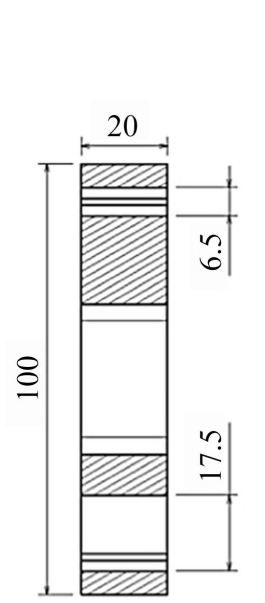

Section view C-C
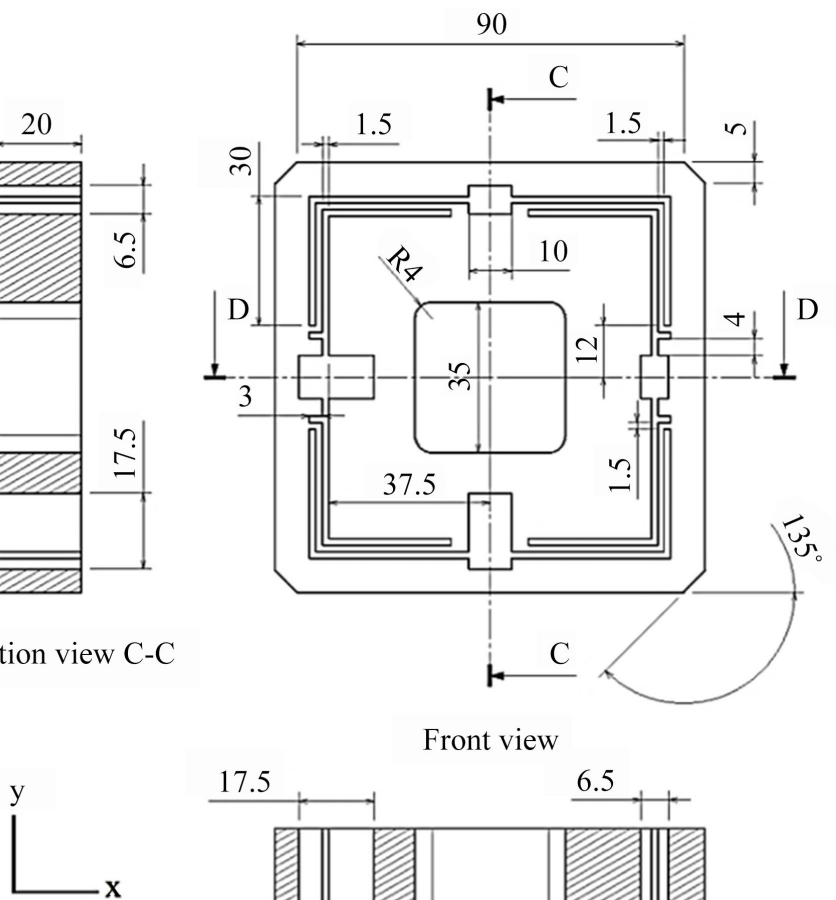

Front view

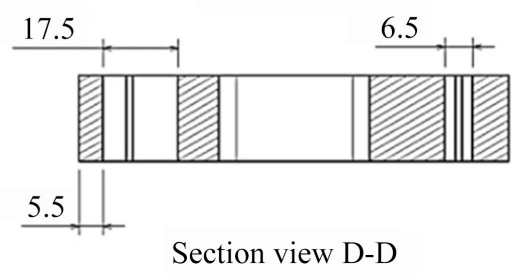

Figure 3. Dimensions of the frame for simulation (all dimensions are in millimeter).
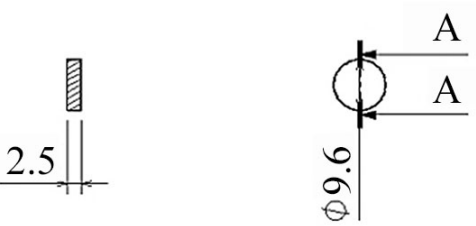

\section{Section view A-A Front view}

Figure 4. Dimensions of the cylindrical piezoelectric disks for simulation (all dimensions are in millimeter). 


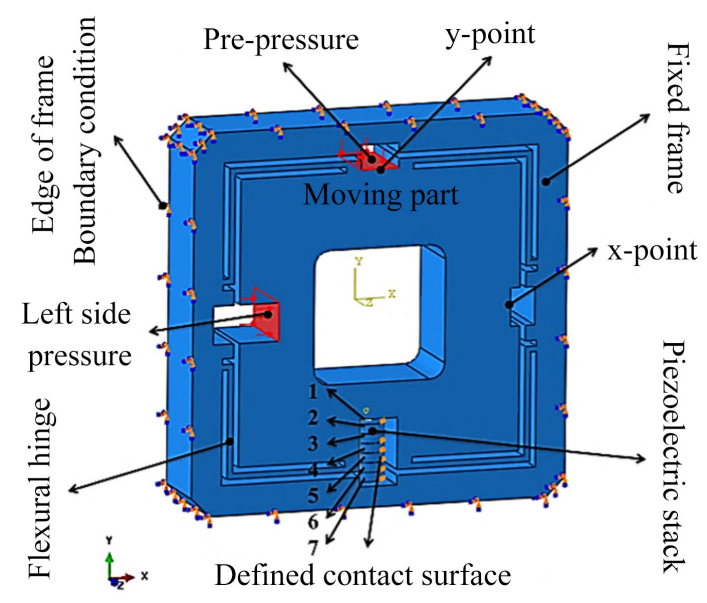

Figure 5. Model of the assembled components including defined contact surfaces, applied boundary conditions and pressure.

Table 1. Mechanical properties of the frame and the cylindrical piezoelectric disk [43] [44] [45].

\begin{tabular}{cccc}
\hline Component & Material type & Young's modulus (GPa) & Poisson's ratio \\
\hline Frame & Aluminium & 68.9 & 0.33 \\
Piezoelectric disk & PICMA` P-885 Ceramic & 36 & 0.34 \\
\hline
\end{tabular}

The surface contact between different piezoelectric elements is modeled through Coulomb's law of Friction. This model can prevent the piezoelectric surfaces from unwanted penetration by selecting the hard contact penetration option in ABAQUS. These surface contacts include contacts of the piezoelectric elements with each other, the contact of piezoelectric disk \#1 with the moving part of the frame, and the contact of the piezoelectric disk \#7 with the fixed part of the frame. Friction coefficients for the three different cases used in this study are tabulated in Table 2. These values have been adopted from the reference [43].

The first, second, and third cases are related to the different friction coefficients between the piezoelectric disks, whereas the friction coefficients between the top and bottom piezoelectric disks and the frame are kept the same for all cases.

Finite element analysis can be used to investigate the effects of different materials for the frame and the piezoelectric ceramics on the cross-coupling effect. The effects of geometrical perturbations, such as changes in the square hole's dimensions inside the frame and the flexural hinges, on the cross-coupling effect, could be investigated.

Therefore, the following cases to this investigation are added:

Fourth case: Changing the material of the frame from Aluminum to Steel with Young's modulus of $210 \mathrm{GPa}$ and Poisson's ratio of 0.3. In this case, the frictional conditions are similar to the first case. 
Table 2. The friction coefficients between the piezoelectric disks with each other, and disk \#1 and disk \#7 with the frame.

\begin{tabular}{cccc}
\hline Friction case & Between piezo disks & $\begin{array}{c}\text { Between piezo disk \#7 } \\
\text { and the fixed frame }\end{array}$ & $\begin{array}{c}\text { Between piezo disk \#1 and } \\
\text { moving part of the frame }\end{array}$ \\
\hline First & 0.1 & 0.7 & 0.7 \\
Second & 0.2 & 0.7 & 0.7 \\
Third & 0.3 & 0.7 & 0.7 \\
\hline
\end{tabular}

Fifth case: Changing the piezoelectric ceramics material with Young's modulus of $11 \mathrm{GPa}$ and Poisson's ratio of 0.34 . In this case, the frictional conditions are similar to the first case.

Sixth case: Changing the geometry of the frame by changing the dimensions of the inner square hole. These changes include changing the length from $35 \mathrm{~mm}$ to $26.25 \mathrm{~mm}$ and the radius corner from $4 \mathrm{~mm}$ to $3 \mathrm{~mm}$, as shown in Figure 6 . In this case, the material properties are similar to Table 1 , and the frictional conditions are similar to the first case.

Seventh case: Changing the frame's geometry by decreasing the thickness of one of the hinges in the y-direction from $1.5 \mathrm{~mm}$ to $1.25 \mathrm{~mm}$, as shown in Figure 7. In this case, the material properties are similar to Table 1 , and the frictional conditions are similar to the first case.

In all cases, all the six degrees of freedom of the frame edges are constrained. Actuation by piezoelectric stacks for the moving part of the frame is modeled with a left-side pressure and pre-pressures in the $\mathrm{x}$ and $\mathrm{y}$ directions, respectively. These pressures are applied in lieu of the voltage applied to the piezoelectric actuators (see Figure 5). Magnitudes of these loadings are adjusted in a way that the same displacements are gained from both the voltage and the pressure excitation methods.

This simulation includes the following four important steps:

1) Pre-pressure loading step;

2) Left side pressure loading step;

3) Pre-pressure unloading step;

4) Left side pressure unloading step.

In other words, the loading process consists of two primary steps, and the third and fourth steps are the unloading process of the model. At the first step, only a pre-pressure with a magnitude of $25 \mathrm{KPa}$ is applied. This pressure is applied smoothly and statically within 0.05 seconds. At the second step, another pressure is applied to the moving frame's left side without changing the prepressure applied in the first step. This pressure is equal to $148 \mathrm{KPa}$, which is applied smoothly and statically within 1 second. So, the loading process takes 1.05 seconds to be completed. In the third step, the pre-pressure is unloaded within 0.05 seconds while no changes are applied to the left side pressure. In the fourth step, the left side pressure is unloaded at 1 second. So, the unloading process, which is completed in the third and fourth steps, takes 1.05 seconds, the same as the loading process, resulting in a total simulation time of 2.1 seconds. Simulation parameters are tabulated in Table 3. Note that due to the low acceleration 
of the system, all simulations are considered to be static.

The type of element used for meshing is C3D8R, an eight-node linear brick element with 24 DOFs. This type of element is capable of traversing shear deformation. The meshing of different parts of the frame, hinges, and the piezoelectric stack is shown in Figure 8.

\section{Results}

The simulation results for the seven cases mentioned in the previous section are discussed here. The simulation results of the first case for the displacement and stress distributions in the stage at the end of loading are shown in Figure 9(a) and Figure 9(b), respectively.

Table 3. Simulation steps.

\begin{tabular}{ccccc}
\hline $\begin{array}{c}\text { Step } \\
\text { number }\end{array}$ & Step & Step time & $\begin{array}{c}\text { Pre-pressure } \\
\text { magnitude (KPa) }\end{array}$ & $\begin{array}{c}\text { Left side pressure } \\
\text { magnitude (KPa) }\end{array}$ \\
\hline 1 & Pre-pressure loading & 0.05 & 25 & 0 \\
2 & Left side pressure loading & 1 & 25 & 148 \\
3 & Pre-pressure unloading & 0.05 & 0 & 148 \\
4 & Left side pressure unloading & 1 & 0 & 0 \\
\hline
\end{tabular}

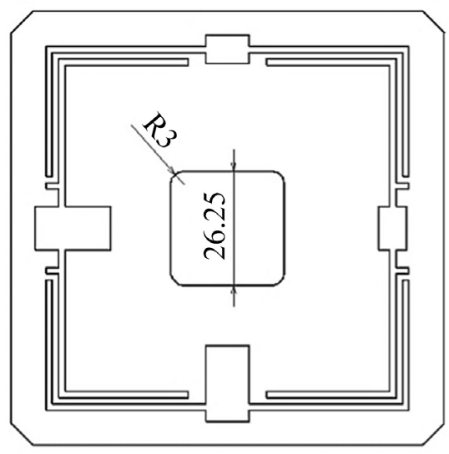

Front view

Figure 6. Decreasing the dimensions of the square hole inside the frame in the sixth case compared to Figure 5 (all dimensions are in millimeter).

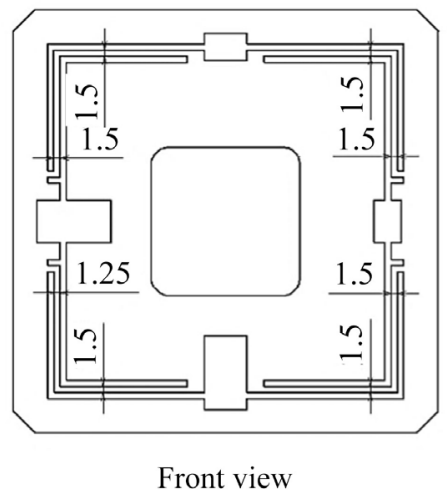

Figure 7. Decreasing the dimensions of the hinge of the frame in the seventh case compared to Figure 5 (all dimensions are in millimeter). 
(a)

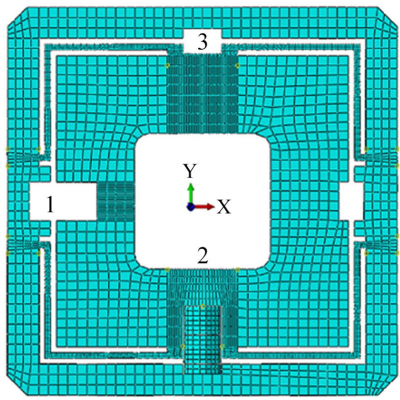

(b)

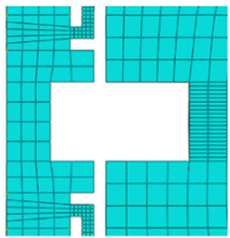

(d)

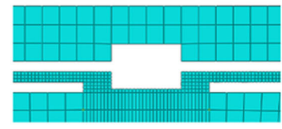

(c)

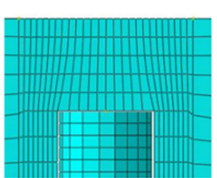

(e)

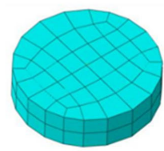

Figure 8. Meshing of: (a) stage; (b) region 1; (c) region 2; (d) region 3; and (e) piezoelectric disk.

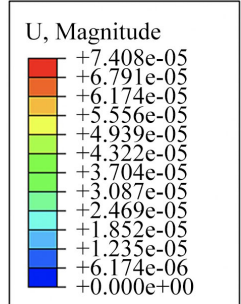

(a)
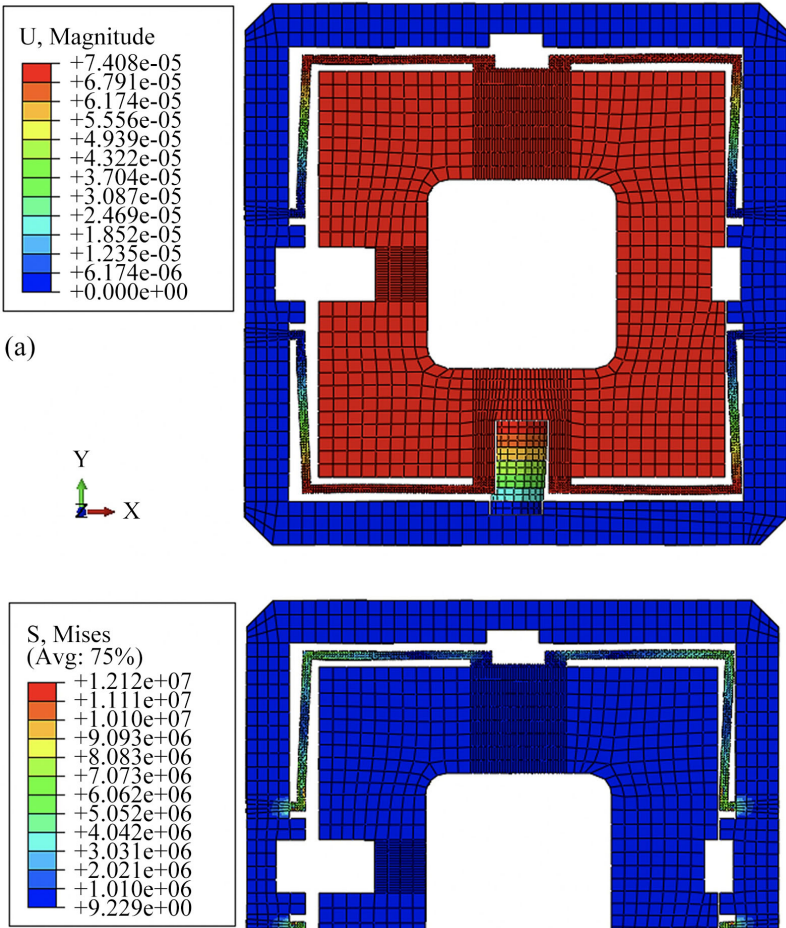

(b)

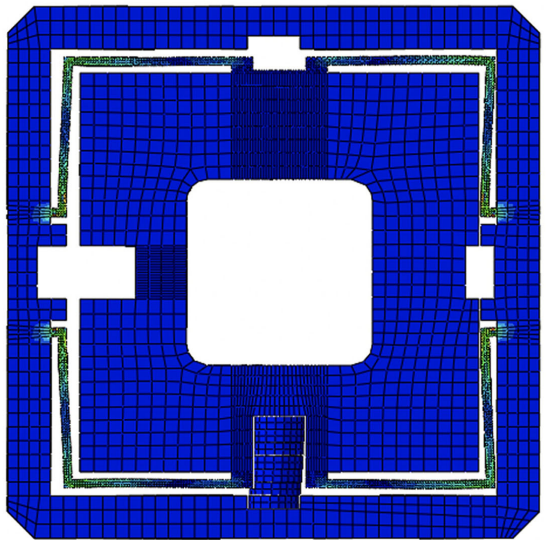

Figure 9. (a) Deformation (meter) and (b) stress distribution (Pa) in the PPNP stage at the end of loading. 
The stress analysis result shows that the flexural hinges bear the highest stress levels, especially at the connection points to the fixed part of the frame. The results of the first case for the mechanical cross-coupling effect are shown in Figure 10. These results are also compared with the experimental data presented earlier in Figure 2. In Figure 10, point D to A represents the first step, and point A to $B$ represents the second step. Hence, point D to B represents the loading process's total time, i.e., 1.05 seconds. Also, point $\mathrm{B}$ to $\mathrm{C}$ represents the third step, and point $C$ to $D$ represents the fourth step. Hence, point B to D represents the total time of unloading, i.e., 1.05 seconds.

Note that the mismatch between the two diagrams in Figure 10 is due to excluding hysteresis and creep models in the simulation. Due to good agreement between the experimental and simulation results, these data and figures are used as a reference for other simulation cases. As seen from Figure 10, by applying the pre-pressure only (first step), the stage moves in the opposite y-direction, but due to the mechanical cross-coupling effect, a small amount of displacement is transferred to the $\mathrm{x}$-direction. In the second step (applying the left side pressure and holding the pre-pressure), the stage moves in both $\mathrm{x}$ and $\mathrm{y}$ directions from point $\mathrm{A}$ to point $\mathrm{B}$ due to the cross-coupling and slipping of the piezoelectric disks on top of each other. At the end of the loading step (Point B), the unloading steps start by removing the pre-pressure in the third step and the left side pressure in the fourth step, respectively. Also, it can be seen in Figure 10 that, due to the low friction coefficient between the piezoelectric disks, and consequently, preventing the stick-slip phenomena from happening, the mechanical cross-coupling effect in all steps remains linear.

The convergence of simulation is a key factor in ensuring the correctness of numerical simulation. To end this, the simulations with different mesh sizes (coarse and fine mesh size) are run and the results are shown in Figure 11. The fine mesh element size is reduced by a factor of 0.1 compared to the coarse mesh.

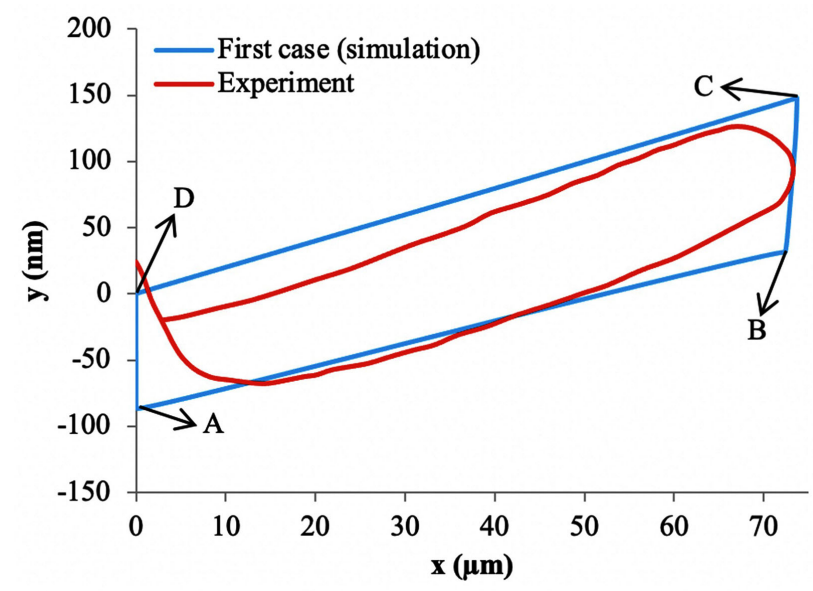

Figure 10. FE simulation and experimental results of the mechanical cross-coupling effect for the first case. 


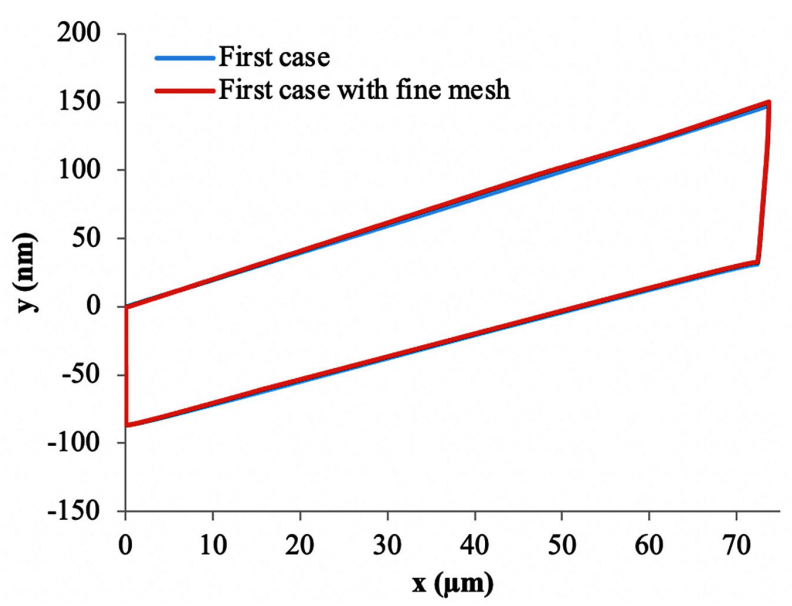

Figure 11. The FE simulation with two different mesh sizes.

In order to evaluate the effect of different friction coefficients, i.e., 0.1, 0.2, and 0.3 , between the piezoelectric disks, the numerical simulations are re-run and the results are plotted in Figure 12. This figure clearly shows that changing the friction coefficient does not have any impact on the displacement of the stage in the first step at Point A, and it moves on a similar path to the first case. That is because no frictional forces were generated in this step. Due to the increasing friction coefficient between the piezoelectric stacks and the friction force emerging from the left side pressure in the second step, the mechanical cross-coupling effect changes from the linear one to two nonlinear and linear sections. The nonlinear section is due to the momentary separation of the piezoelectric disks from each other (stick-slip effect) and the rotation of the stack due to powerful frictional forces between piezoelectric disks. Following these phenomena, a sudden change of displacement occurs in the y-direction. It causes Point $\mathrm{B}$ to change its position to another point. In other words, it can be seen that by increasing the friction coefficient, a different displacement path happens in the PPNP stage. This change of displacement leads to changing the stage's behavior from linear to nonlinear because of higher frictional forces. More disordering behavior is caused by increasing the friction coefficient. After the initial nonlinear response, the stage's response becomes linear due to the piezoelectric disks' steady slip on each other. In the third step, by unloading the pre-pressure, the stage moves to Point $\mathrm{C}$, and in the fourth step, by unloading the left side pressure, the stage returns to its initial position at Point $\mathrm{D}$ on a similar path to the first case.

In order to understand the effects of the frame's material stiffness, the FEA simulations for two different frames Young's modulus values are run and the results are plotted in Figure 13.

As can be seen from Figure 13, increasing Young's modulus of the frame leads to decreasing the displacement of the stage in both directions, while the friction coefficient is fixed like the first case. Increasing Young's modulus causes 
the stiffness of the stage to increase. Note that the stiffness of the stage is proportional to that of the hinges. Lin and Chen [46] showed that the stiffness of the hinge $K_{\text {hinge }}$ is proportional to Young's modulus and the third power of the hinge thickness in the XY plane:

$$
K_{\text {stage }} \propto K_{\text {hinge }} \propto E d^{3}
$$

where $E$ is Young's modulus and $d$ is the thickness of the hinge in the XY plane.

Therefore, due to an identical increase of the stage stiffness in both directions, the cross-coupling effect does not undergo any changes. This result is in good agreement with the experimental results.

To evaluate the effects of the piezoelectric material's stiffness on the mechanical cross-coupling effect, the FEA simulation for two different piezoelectric Young's modulus values are rerun and the results are plotted in Figure 14.

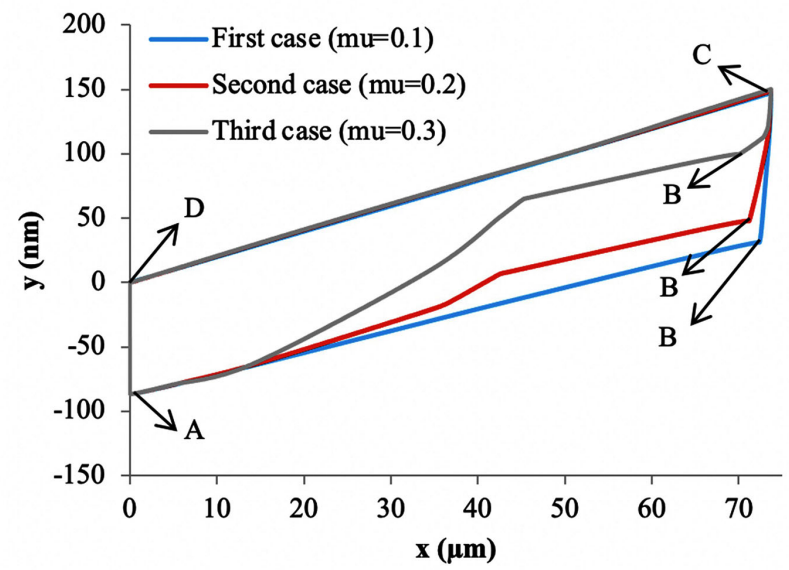

Figure 12. The effect of the friction coefficient of the piezoelectric disks on the mechanical cross-coupling effect (comparison between cases 1, 2, and 3).

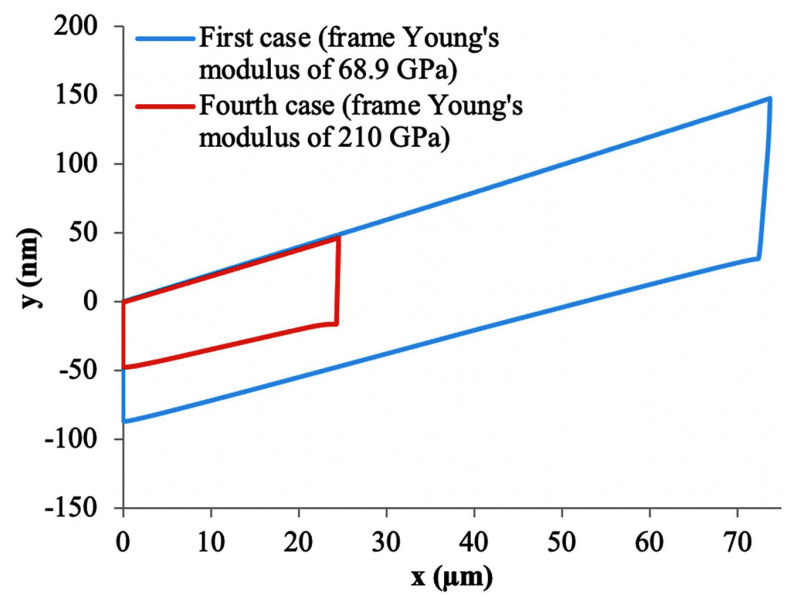

Figure 13. The effects of Young's modulus of the frame material on the mechanical cross-coupling effect (comparison between Case 1 and 4). 


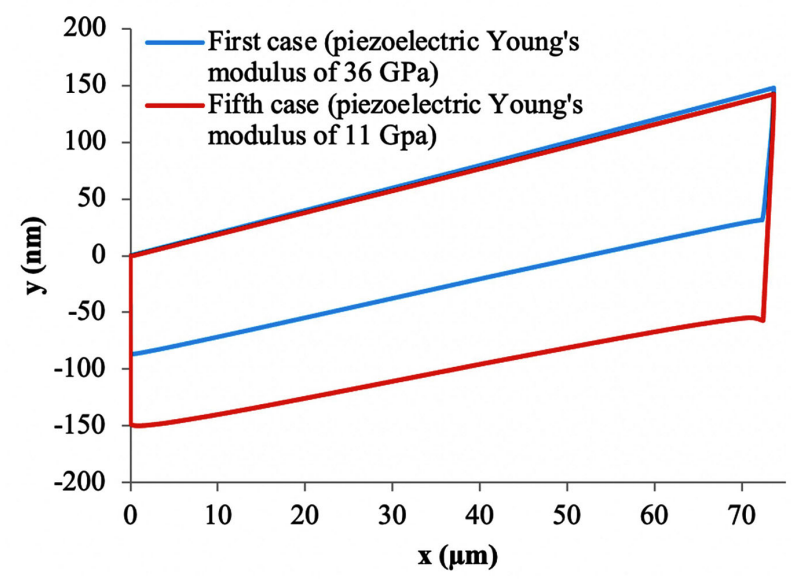

Figure 14. The effects of Young's modulus of the piezoelectric material on the mechanical cross-coupling effect (comparison between Case 1 and 5).

It can be seen from Figure 14 that decreasing Young's modulus of the piezoelectric stacks does not affect the stiffness of the stage in either of directions and consequently, the cross-coupling effect and the displacement in the $\mathrm{x}$-direction do not change. Note that for both cases, the friction coefficient is similar to the first case. It is evident from this figure that decreasing piezoelectric stiffness causes the displacement of the stage to decrease in the y-direction. As a result, it can be concluded that changing the frame's material properties or the piezoelectric stacks does not have any impacts on the mechanical cross-coupling effect.

In order to investigate the impacts of changing the stage's dimension on the mechanical cross-coupling effect, the FEA simulation for two cases with different frame and hinge geometries (sixth and seventh case) are re-run and the results are plotted in Figure 15 and Figure 16.

It can be seen from Figure 15 that by decreasing the dimensions of the inner hole and maintaining the material properties and frictional conditions of the first case, the stage's stiffness does not change in the $\mathrm{x}$-direction, but it increases in the $\mathrm{y}$-direction. Hence, as the stage has no displacement in the $\mathrm{x}$-direction comparing to the first case, its displacement decreases in the y-direction. In total, the mechanical cross-coupling effect decreases.

It can be concluded from Figure 16 that by decreasing the hinges' thickness in the $y$-direction, the stiffness of the hinge decreases as well. However, the stiffness of the stage in the $\mathrm{y}$-direction decreases more than that in the $\mathrm{x}$-direction. Hence, the cross-coupling effect is amplified. The amplification of the cross-coupling effect is due to the decrease in the stage's stiffness, which can also be concluded from Equation (1).

By analyzing Figure 15 and Figure 16, it is evident that one of the other ways to change the mechanical cross-coupling effect between the two axes is changing the stage's geometry. In other words, in some cases where the stiffness ratio between the two axes is changed, the mechanical cross-coupling effect is altered. 


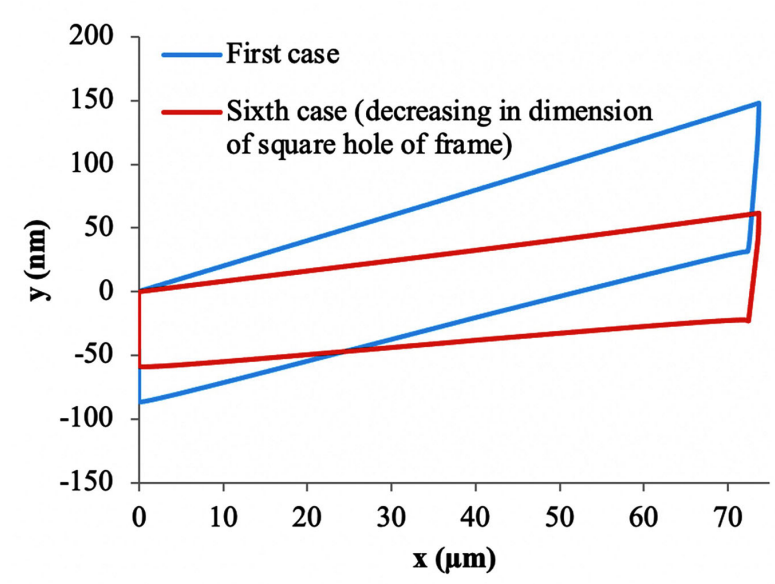

Figure 15. The effect of decreasing the dimension of square hole of the frame on the mechanical cross-coupling effect (comparison between Case 1 and 6).

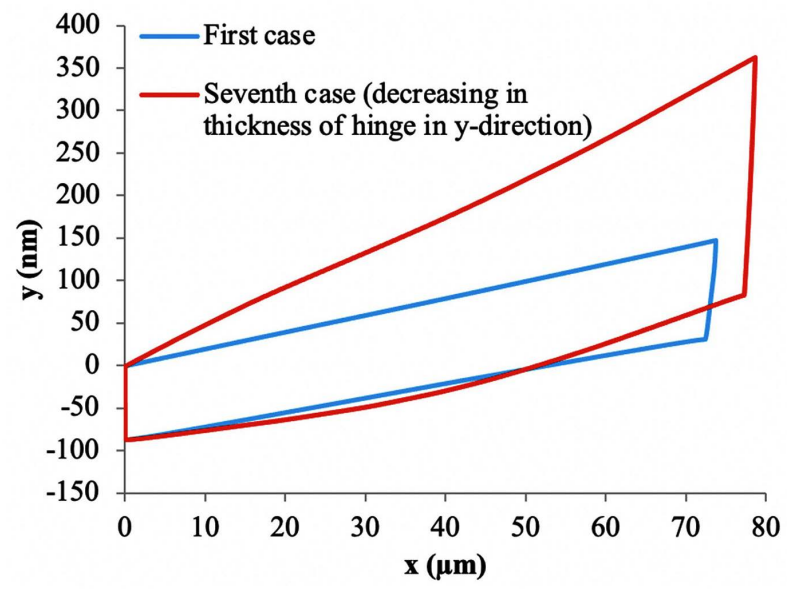

Figure 16. The effect of decreasing the hinge's thickness in the $y$-direction on the mechanical cross-coupling effect (comparison between Case 1 and 7).

\section{Conclusions}

In this study, FEA simulations are carried out to investigate the effects of different mechanical properties, frictional behavior, and geometry of parallel piezoflexural nanopositioning (PPNP) stages on the mechanical cross-coupling effect between their different axes. In all simulation and experimental trials, the effects of inertia and damping at low frequencies were ignored. The stress distribution in the stage shows that the flexural hinges have the highest stress level, especially at the connection points to the fixed part of the frame. Also, surveying the influence of friction coefficient between piezoelectric disks shows that the piezoelectric disks slip on each other at low friction coefficients, resulting in a linear cross-coupling behavior. Increasing the friction coefficient leads to a sudden change in the displacement of the stage in the y-direction when the excitation forces are present in the $\mathrm{x}$-direction only, and the piezoelectric disks undergo a 
stick-slip frictional behavior. This sticking leads to some degree of rotation in the piezoelectric disks, resulting in a nonlinear cross-coupling behavior. Another observation shows that changing the frame's material causes the stage's stiffness to be altered in both directions. Hence, changing the material of the frame has no impact on the cross-coupling effect.

Moreover, changing the piezoelectric disks' material does not cause the crosscoupling effect to be changed, as it does not affect the stage's stiffness. Different geometries of the stage are also considered by changing both the dimensions of the inner hole of the stage and the hinges' thickness. The result is that changing the dimensions of the inner hole of the stage only causes the stiffness to be changed in the y-direction, and consequently, the cross-coupling effect is changed. FEA simulations also show that the proportion of stiffness between two axes is changed by choosing a different thickness for the hinge. This consequently causes the mechanical cross-coupling effect to be changed. Finally, the main parameters that affect the mechanical cross-coupling are the combination of slip and rotation of the piezoelectric disks concurrent with a change in the stage's geometry. The results obtained in this paper can provide a better insight into the mechanics of the PPNP stages for potential improvement of the cross-coupling effect in the product design and development phase.

\section{Acknowledgements}

The first author would like to sincerely thank Dr. Peiman Mosaddegh, Dr. Saeed Bashash, and Dr. Nader Jalili. This work would not have been possible without their scientific supports.

\section{Conflicts of Interest}

The authors declare no conflicts of interest regarding the publication of this paper.

\section{References}

[1] Meli, F. and Thalmann, R. (1998) Long-Range AFM Profiler Used for Accurate Pitch Measurements. Measurement Science and Technology, 9, 1087-1092. https://doi.org/10.1088/0957-0233/9/7/014

[2] Bonnail, N., Tonneau, D., Jandard, F., Capolino, G. and Dallaporta, H. (2004) Variable Structure Control of a Piezoelectric Actuator for a Scanning Tunneling Microscope. IEEE Transactions on Industrial Electronics, 51, 354-363. https://doi.org/10.1109/TIE.2004.825266

[3] Binnig, G. and Rohrer, H. (1983) Scanning Tunneling Microscopy. Surface Science, 126, 236-244. https://doi.org/10.1016/0039-6028(83)90716-1

[4] Binnig, G. and Smith, D.P.E. (1986) Single-Tube Three-Dimensional Scanner for Scanning Tunneling Microscopy. Review of Scientific Instruments, 57, 1688-1689. https://doi.org/10.1063/1.1139196

[5] Hosseini, R., Zargar, O. and Hamedi, M. (2018) Improving Power Density of Piezoelectric Vibration-Based Energy Scavengers. Journal of Solid Mechanics, 10, 98 109. http://jsm.iau-arak.ac.ir/article_539696.html 
[6] Rahmati, A.H., Yang, S., Bauer, S. and Sharma, P. (2019) Nonlinear Bending Deformation of Soft Electrets and Prospects for Engineering Flexoelectricity and Transverse (d 31) Piezoelectricity. Soft Matter, 15, 127-148.

https://doi.org/10.1039/C8SM01664J

[7] Aryafar, M., Hamedi, M. and Ganjeh, M.M. (2015) A Novel Temperature Compensated Piezoresistive Pressure Sensor. Measurement, 63, 25-29.

https://doi.org/10.1016/j.measurement.2014.11.032

[8] Onsorynezhad, S., Abedini, A. and Wang, F. (2020) Parametric Optimization of a Frequency-Up-Conversion Piezoelectric Harvester via Discontinuous Analysis. Journal of Vibration and Control, 26, 1241-1252. https://doi.org/10.1177/1077546319894797

[9] Pasharavesh, A., Moheimani, R. and Dalir, H. (2020) Performance Analysis of an Electromagnetically Coupled Piezoelectric Energy Scavenger. Energies, 13, 845. https://doi.org/10.3390/en13040845

[10] Ahmadian, A. (2020) Design and Fabrication of High Capacity Lithium-Ion Batteries Using Electro-Spun Graphene Modified Vanadium Pentoxide Cathodes. Diss., Purdue University Graduate School, Indianapolis, USA.

[11] Derakhshan, R., Ahmadian, M.T. and Firoozbakhsh, K. (2018) Pull-In Criteria of a Nonclassical Microbeam under Electric Field Using Homotopy Method. Statistical Center of Iran, 25, 175-185.

[12] Hashemi, T.S., Iravani, M. and Farahmand, F. (2020) Effects of High Tibia Osteotomy on Knee Ligamentous Forces during Gait: A Finite Element Study. 202027 th National and 5th International Iranian Conference on Biomedical Engineering (ICBME), Tehran, Iran, 26-27 November 2020, 182-186.

https://doi.org/10.1109/ICBME51989.2020.9319433

[13] Hoursan, H., Farahmand, F. and Ahmadian, M.T. (2021) Effect of Axonal Fiber Architecture on Mechanical Heterogeneity of the White Matter-A Statistical Micromechanical Model. Computer Methods in Biomechanics and Biomedical Engineering, 1-13. https://doi.org/10.1080/10255842.2021.1927000

[14] Shafiee, A., Ahmadian, M.T., Hoursan, H. and Hoviat Talab, M. (2015) Effect of Linear and Rotational Acceleration on Human Brain. Modares Mechanical Engineering, 15, 248-260.

[15] Abderezaei, J., et al. (2019) Nonlinear Dynamical Behavior of the Deep White Matter during Head Impact. Physical Review Applied, 12, Article ID: 014058. https://doi.org/10.1103/PhysRevApplied.12.014058

[16] Shafiee, A., Ahmadian, M.T. and Hoviattalab, M. (2016) Mechanical Characterization of Brain Tissue in Compression. ASME 2016 International Design Engineering Technical Conferences and Computers and Information in Engineering Conference, Vol. 50138, V003T11A001.

[17] Shafiee, A., Ahmadian, M.T. and Hoviattalab, M. (2016) Traumatic Brain Injury Caused by $+\mathrm{Gz}$ Acceleration. ASME 2016 International Design Engineering Technical Conferences and Computers and Information in Engineering Conference, Vol. 50138, V003T11A002.

[18] Brown, M.S., Mendoza, M., Chavoshnejad, P., Razavi, M.J., Mahler, G.J. and Koh, A. (2020) Biofluid-Permeable Electronics: Electronic-ECM: A Permeable Microporous Elastomer for an Advanced Bio-Integrated Continuous Sensing Platform. Advanced Materials Technologies, 5, Article ID: 2070043.

https://doi.org/10.1002/admt.202070043

[19] Ardestani, M.M., ZhenXian, C., Noori-Dokht, H., Moazen, M. and Jin, Z. (2019) 
Computational Analysis of Knee Joint Stability Following Total Knee Arthroplasty. Journal of Biomechanics, 86, 17-26. https://doi.org/10.1016/j.jbiomech.2019.01.029

[20] Shafiee, A., Ahmadian, M.T., Hoursan, H. and Hoviat Talab, M. (2014) TwoDimensional Modeling and Analysis of the Effect of Linear Acceleration on Brain Strain Field in Traumatic Brain Injury. 22nd Annual International Conference on Mechanical Engineering-ISME, Ahvaz, 22-24 April 2014, 22-29.

[21] Akbari, A. and Chen, J. (2019) Peak Load Distribution of Vibrational Devices for Accelerating Tooth Movement. 2019 IADR/AADR/CADR General Session \& Exhibition, Vancouver, 19-22 Jun 2019, 1-4.

[22] Akbari, A. (2019) The Quantification of Force Distribution of a Vibrational Device for Accelerating Tooth Movement.

[23] Jiang, F., Roberts, W.E., Liu, Y., Shafiee, A. and Chen, J. (2020) Mechanical Environment for Lower Canine T-Loop Retraction Compared to En-Masse Space Closure with a Power-Arm Attached to Either the Canine Bracket or the Archwire. The Angle Orthodontist, 90, 801-810. https://doi.org/10.2319/050120-377.1

[24] Khezrloo, A., Tayebi, M., Shafiee, A. and Aghaie, A. (2021) Evaluation of Compressive and Split Tensile Strength of Slag Based Aluminosilicate Geopolymer Reinforced by Waste Polymeric Materials Using Taguchi Method. Materials Research Express, 8, 25504. https://doi.org/10.1088/2053-1591/abe101

[25] Kazemi, A. and Yang, S. (2019) Atomistic Study of the Effect of Magnesium Dopants on the Strength of Nanocrystalline Aluminum. JOM, 71, 1209-1214. https://doi.org/10.1007/s11837-019-03373-3

[26] Shahverdi Moghaddam, H., Kothare, A., Keshavanarayana, S.R., Yang, C.C. and Horner, A.L. (2017) Experimental and Numerical Study on the Mechanical Response of Fiberglass/Phenolic Honeycomb Core under Uniaxial In-Plane Loading.

[27] Rezazadeh Kalehbasti, P. and Dolatshahi, K.M. (2018) Two Novel Shear Fuses in Moment Resisting Frames. Journal of Constructional Steel Research, 144, 198-210. https://doi.org/10.1016/j.jcsr.2018.01.026

[28] Akbar, M., Ahmadian, A., Sadeghi, M.J., Ahmadian, A., Farahmand, F. and Sarkar, S. (2020) Parseh Intelligent Surgical System. Robotic Guide for Brain Biopsy. US Patent 10, 555,784.

[29] Shamloo, A. and Boodaghi, M. (2018) Design and Simulation of a Microfluidic Device for Acoustic Cell Separation. Ultrasonics, 84, 234-243.

https://doi.org/10.1016/j.ultras.2017.11.009

[30] Hadad, S., Mut, F., Chung, B.J., Roa, J.A., Robertson, A.M., Hasan, D.M., Samaniego, E.A. and Cebral, J.R. (2020) Regional Aneurysm Wall Enhancement Is Affected by Local Hemodynamics: A 7T MRI Study. American Journal of Neuroradiology, 42, 464-470. https://doi.org/10.3174/ajnr.A6927

[31] Alidoost, M. and Reza Pishevar, A. (2018) On Importance of the Surface Charge Transport Equation in Numerical Simulation of Drop Deformation in a Direct Current Field. Journal of Fluids Engineering, 140, Article ID: 121201. https://doi.org/10.1115/1.4040301

[32] Van de Werken, N., Hurley, J., Khanbolouki, P., Sarvestani, A.N., Tamijani, A.Y. and Tehrani, M. (2019) Design Considerations and Modeling of Fiber Reinforced 3D Printed Parts. Composites Part B: Engineering, 160, 684-692. https://doi.org/10.1016/j.compositesb.2018.12.094

[33] Nasiri, R., Shamloo, A., Akbari, J., Tebon, P., Dokmeci, M.R. and Ahadian, S. (2020) Design and Simulation of an Integrated Centrifugal Microfluidic Device for CTCs Separation and Cell Lysis. Micromachines, 11, 699. 
https://doi.org/10.3390/mi11070699

[34] Elmustafa, A. and Lagally, M.G. (2001) Flexural-Hinge Guided Motion Nanopositioner Stage for Precision Machining: Finite Element Simulations. Precision Engineering, 25, 77-81. https://doi.org/10.1016/S0141-6359(00)00058-1

[35] Sun, L.N., Ma, L., Rong, W.B. and Gao, Y. (2006) Design and Analysis on a 2-DOF Nanopositioning Stage. Optics and Precision Engineering, 14, 406-411.

[36] Li, Y. and Xu, Q. (2010) A Novel Piezoactuated XY Stage with Parallel, Decoupled, and Stacked Flexure Structure for Micro-/Nanopositioning. IEEE Transactions on Industrial Electronics, 58, 3601-3615. https://doi.org/10.1109/TIE.2010.2084972

[37] Shi, H., Kim, Y. and She, Y. (2015) Design of a Parallel Kinematic MEMS XY Nanopositioner. 2015 IEEE International Conference on Robotics and Biomimetics (ROBIO), Zhuhai, 6-9 December 2015, 1973-1978.

https://doi.org/10.1109/ROBIO.2015.7419062

[38] Nagel, W.S. and Leangy, K.K. (2017) Design of a Dual-Stage, Three-Axis Hybrid Parallel-Serial-Kinematic Nanopositioner with Mechanically Mitigated Cross-Coupling. 2017 IEEE International Conference on Advanced Intelligent Mechatronics (AIM), Munich, 3-7 July 2017, 706-711. https://doi.org/10.1109/AIM.2017.8014100

[39] Zhang, H.-T. et al. (2018) Distributed Hammerstein Modeling for Cross-Coupling Effect of Multiaxis Piezoelectric Micropositioning Stages. IEEE/ ASME Transactions on Mechatronics, 23, 2794-2804. https://doi.org/10.1109/TMECH.2018.2870864

[40] Kang, S., Wu, H., Yu, S., Li, Y., Yang, X. and Yao, J. (2020) Modeling and Control of a Six-Axis Parallel Piezo-Flexural Micropositioning Stage with Cross-Coupling Hysteresis Nonlinearities. 2020 IEEE/ ASME International Conference on Advanced Intelligent Mechatronics (AIM), Boston, 6-9 July2020, 1350-1355. https://doi.org/10.1109/AIM43001.2020.9158988

[41] Bashash, S. (2008) Modeling and Control of Piezoactive Micro and Nano Systems.

[42] Bashash, S. and Jalili, N. (2009) Robust Adaptive Control of Coupled Parallel PiezoFlexural Nanopositioning Stages. IEEE/ASME Transactions on Mechatronics, 14, 11-20. https://doi.org/10.1109/TMECH.2008.2006501

[43] Positioning with Piezo Systems Catalog (2021). https://static.pi-usa.us/fileadmin/user_upload/pi_us/files/catalogs/PI-Positioning-w ith-Piezo-Systems_catalog.pdf

[44] Shafiee, A., Mosadegh, P., Bashash, S. and Jalili, N. (2014) Study of Cross-Coupling Effect in Piezo-Flexural Nanopositioning Stages. Modares Mechanical Engineering, 14, 1-8.

[45] Shafiee, A., Mosadegh, P., Bashash, S. and Jalil, N. (2013) Numerical Simulation of Positioning in Piezoelectrically-Actuated Nano Stages. 21 st Annual International Conference on Mechanical Engineering-ISME, Tehran, 7-9 May 2013, 8-12.

[46] Lin, C.-Y. and Chen, P.-Y. (2011) Precision Tracking Control of a Biaxial Piezo Stage Using Repetitive Control and Double-Feed forward Compensation. Mechatronics, 21, 239-249. https://doi.org/10.1016/j.mechatronics.2010.11.002 